\title{
Investigating Content Selection for Language Generation using Machine Learning
}

\author{
Colin Kelly \\ Computer Laboratory \\ University of Cambridge \\ $15 \mathrm{JJ}$ Thomson Avenue \\ Cambridge, UK \\ Ann Copestake \\ Computer Laboratory \\ University of Cambridge \\ $15 \mathrm{JJ}$ Thomson Avenue \\ Cambridge, UK \\ Nikiforos Karamanis \\ Department of Computer Science \\ Trinity College Dublin \\ Dublin 2 \\ Ireland \\ \{colin.kelly, ann.copestake, nikiforos.karamanis\}@cl.cam.ac.uk
}

\begin{abstract}
The content selection component of a natural language generation system decides which information should be communicated in its output. We use information from reports on the game of cricket. We first describe a simple factoid-to-text alignment algorithm then treat content selection as a collective classification problem and demonstrate that simple 'grouping' of statistics at various levels of granularity yields substantially improved results over a probabilistic baseline. We additionally show that holding back of specific types of input data, and linking database structures with commonality further increase performance.
\end{abstract}

\section{Introduction}

Content selection is the task executed by a natural language generation (NLG) system of deciding, given a knowledge-base, which subset of the information available should be conveyed in the generated document (Reiter and Dale, 2000).

Consider the task of generating a cricket match report, given the scorecard for that match. Such a scorecard would typically contain a large number of statistics pertaining to the game as a whole as well as individual players (e.g. see Figure 1). Our aim is to identify which statistics should be selected by the NLG system.

Much work has been done in the field of content selection, in a diverse range of domains e.g. weather forecasts (Coch, 1998). Approaches are usually domain specific and predominantly based on structured tables of well-defined input data.

Duboue and McKeown (2003) attempted a statistical approach to content selection using a substantial corpus of biographical summaries paired with selected content, where they extracted rules and patterns linking the two. They then used machine learning to ascertain what was relevant.

Barzilay and Lapata (2005) extended this approach but applying it to a sports domain (American football), similarly viewing content selection as a classification task and additionally taking account of contextual dependencies between data, and found that this improved results compared to a content-agnostic baseline. We aim throughout to extend and improve upon Barzilay and Lapata's methods.

We emphasise that content selection through statistical machine learning is a relatively new area - approaches prior to Duboue and McKeown's are, in principle, much less portable - and as such there is not an enormous body of work to build upon.

This work offers a novel algorithm for data-totext alignment, presents a new 'grouping' method for sharing knowledge across similar but distinct learning instances and shows that holding back certain data from the machine learner, and reintroducing it later on can improve results.

\section{Data Acquisition \& Alignment}

We first must obtain appropriately aligned cricket data, for the purposes of machine learning.

Our data comes from the online Wisden almanack (Cricinfo, 2007), which we used to download 133 match report/scorecard pairs. We employed an HTML parser to extract the main text from the match report webpage, and the match data-tables from the scorecard webpage. An example scorecard can be found in Figure $1^{1}$.

\footnotetext{
${ }^{1}$ Cricket is a bat-and-ball sport, contested by two opposing teams of eleven players. Each side's objective is to score more 'runs' than their opponents. An 'innings' refers to the collective performance of the batting team, and (usually) ends when all eleven players have batted.

In Figure 1, in the batting section R stands for 'runs made', $\mathrm{M}$ for 'minutes played on the field', $\mathrm{B}$ for 'number of balls faced'. $4 \mathrm{~s}$ and $6 \mathrm{~s}$ are set numbers of runs awarded for hitting balls that reach the boundary. SR is the number of runs per 100 balls. In the bowling section, $\mathrm{O}$ stands for 'overs
} 
Result India won by 63 runs

\begin{tabular}{llcccccc}
\hline India innings & (50 overs maximum) & R & M & B & 4s & 6s & SR \\
\hline SC Ganguly* & run out (Silva/Sangakarra $\dagger$ ) & 9 & 37 & 19 & 2 & 0 & 47.36 \\
V Sehwag & run out (Fernando) & 39 & 61 & 40 & 6 & 0 & 97.50 \\
D Mongia & b Samaraweera & 48 & 91 & 63 & 6 & 0 & 76.19 \\
SR Tendulkar & c Chandana b Vaas & 113 & 141 & 102 & 12 & 1 & 110.78 \\
$\ldots$ & & & & & & & \\
Extras & (lb 6, w 12, nb 7) & $\mathbf{2 5}$ & & & & & \\
Total & (all out; 50 overs; 223 mins) & $\mathbf{3 0 4}$ & & & & & \\
\hline
\end{tabular}

Fall of wickets 1-32 (Ganguly, $6.5 \mathrm{ov}$ ), 2-73 (Sehwag, $11.2 \mathrm{ov}$ ), 3-172 (Mongia, 27.4 ov), 4-199 (Dravid, 32.1 ov), ..., 10-304 (Nehra, 49.6 ov)

\begin{tabular}{lcccccr}
\hline Bowling & O & M & R & W & Econ & \\
\hline WPUJC Vaas & 10 & 1 & 64 & 1 & 6.40 & $(2 \mathrm{w})$ \\
DNT Zoysa & 10 & 0 & 66 & 1 & 6.60 & $(6 \mathrm{nb}, 2 \mathrm{w})$ \\
․ & & & & & & \\
TT Samaraweera & 8 & 0 & 39 & 2 & 4.87 & $(2 \mathrm{w})$ \\
\hline
\end{tabular}

Figure 1: Statistics in a typical cricket scorecard.

\subsection{Report Alignment}

We use a supervised method to train our data, and thus need to find all 'links' between the scorecard and match report. We execute this alignment by first creating tags with tag attributes according to the common structure of the scorecards, and tag values according to the data within a particular scorecard. We then attempt to automatically align the values of those tags with factoids, single pieces of information found in the report.

For example, from Figure 1 the fact that Tendulkar was the fourth player to bat on the first team is captured by constructing a tag with tag attribute team1_player4, and tag value 'SR Tendulkar'. The fact he achieved 113 runs is encapsulated by another tag, with tag attribute as team1_player $4 R$ and tag value as ' 113 '. Then if the report contained the phrase 'Tendulkar made 113 off 102 balls' we would hope to match the 'Tendulkar' factoid with our tag value 'SR Tendulkar', the '113' factoid with our tag value '113' and replace both factoids with their respective tag attributes, in this case team1_player4 and team1_player4_R respectively. Similar methods for this problem have been employed by Barzilay and Lapata (2005) and Duboue and McKeown (2003).

The basic idea behind our 6-step process for alignment is that we align those factoids we are

bowled', $\mathrm{M}$ for 'maiden overs', $\mathrm{R}$ for 'runs conceded' and $\mathrm{W}$ for 'wickets taken'. Econ is 'economy rate', or number of runs per over.

It is important to note that Figure 1 omits the opposing team's innings (comprising new instances of the 'Batting', 'Fall of Wickets' and 'Bowling' sections), and some additional statistics found at the bottom of the scorecard. most certain of first. The main obstacle we face when aligning is the large incidence of repeated numbers occurring within the scorecard, as this would imply we have multiple, different tags all with the same tag values. It is wholly possible (and quite typical) that single figures will be repeated many times within a single scorecard ${ }^{2}$.

Therefore it would be advantageous for us to have some means to differentiate amongst tags, and hopefully select the correct tag when encountering a factoid which corresponds to repeated tag values. Our algorithm is as follows:

Preprocessing We began by converting all verbalised numbers to their cardinal equivalents, e.g. 'one', 'two' to ' 1 ', ' 2 ', and selected instances of 'a' into ' 1 '.

Proper Nouns In the first round of tagging we attempt to match proper names from the scorecard with strings within the report. Additionally, we maintain a list of all players referenced thus far.

Player-Relevant Details Using the list of players we have accumulated, we search the report for matches on tag values relating to only those players. This step was based on the assumption that a factoid about a specific player is unlikely to appear unless that player has been named.

Non-Player-Relevant Details The next stage involves attempting to match factoids to tag values whose attributes don't refer to a particular player e.g., more general match information as well as team statistics.

\footnotetext{
${ }^{2}$ For example in Figure 1 we can see the number 6 appearing four times: twice as the number of $4 \mathrm{~s}$ for two different players, once as an $l b$ statistic and once as an $n b$ statistic.
} 
Anchor-Based Matching We next use surrounding text anchor-based matching: for example, if a sentence contains the string 'he bowled for 3 overs' we will preferentially attempt to match the factoid ' 3 ' with tag values from tags which we know refer to overs.

Remaining Matches The final step acts as our 'catch-all' - we proceed through all remaining words in the report and try to match each potential factoid with the first (if any) tag found whose tag value is the same.

\subsection{Evaluation}

The output of our program is the original text with all aligned figures and strings (factoids) replaced with their corresponding tag attributes. We can see an extract from an aligned report in Figure 2 where we show the aligned factoids in bold, and their corresponding tag attributes in italics. We also note at this point that much of commentary shown does not in fact appear in the scorecard, and therefore additional knowledge sources would typically be required to generate a full match report - this is beyond the scope of our paper, but Robin (1995) attempts to deal with this problem in the domain of basketball using revision-based techniques for including additional content.

We asked a domain expert to evaluate five of our aligned match reports - he did this by creating his own 'gold standard' for each report, a list of aligned tags. Compared to our automatically aligned tags, we obtained $79.0 \%$ average precision, $75.8 \%$ average recall and a mean $\mathrm{F}$ of $77.0 \%$.

\section{Categorization}

We are using the methods of Barzilay and Lapata (henceforth B\&L) as our starting point, so we describe what we did to emulate and extend them.

\subsection{Barzilay and Lapata's Method}

B\&L's corpus was composed of a relational database of football statistics. Within the database were multiple tables, which we will refer to as 'categories' (actions within a game, e.g. touchdowns and fumbles). Each category was composed of 'groups' (the rows within a category table), with each row referring to a distinct player, and each column referring to different types of action within that category ('attributes').

B\&L's technique for the purposes of the machine learning was to assign a ' 1 ' or ' 0 ' to each
NatWest Series (series), match 9 (team1_player $\left.1 \_R\right)$

India v Sri Lanka (matchtitle)

At Bristol (venue_town), July 11 (date) (day/night (daynight)).

India (team1) won by 63 runs (winmethod).

India (team1) 5 (team1_points) pts.

Toss: India (team1).

The highlight of a meaningless match was a sublime innings from Tendulkar (team1_player4), who resumed his fleeting love affair with Nevil Road to the delight of a flag-waving crowd. On India (teaml)'s only other visit to Bristol (venue_town), for a World Cup game in 1999 against Kenya, Tendulkar (team1_player4) had creamed an unbeaten 140, and this time he drove with elan to make 113 (team1_player4_R) off just 102 (team1_player4_B) balls with 12 (team1_player4_4s) fours and a (team1_player4_6s) six.

Figure 2: Aligned match report extract

row, where a row would receive the value ' 1 ' if one or more of the entries in the row was verbalised in the report. In the context of our data we could apply a similar division, for example, by constructing a category entitled 'Batting' with attributes (columns) 'Runs', 'Balls', 'Minutes', '4s' and ' 6 s' etc., and rows corresponding to players. In this case a group within that category would correspond to one line of the 'Innings' table in Figure 1.

We note that $\mathrm{B} \& \mathrm{~L}$ were selecting content on a row basis, while we are aiming to select individual tag attributes (i.e., specific row/column cell references) within the categories, a more difficult task. We discuss this further in Section 6.

The technique above allows the machine learning algorithm to be aware that different statistics are semantically related - i.e., each group within a category contains the same 'type' of information. We therefore think this is a logical starting point for our work, and we aim to expand upon it.

\subsection{Classifying Tags}

The key step was deciding upon an appropriate division of our scorecard into various categories and the groups for each category in the style of B\&L. As can be seen from Figure 1 our input information is a mixture of structured (e.g. Bowling, Batting sections), semi-structured (Fall of Wickets section) and almost unstructured (Result) information. This is somewhat unlike B\&L's data, which was fully structured in database form. We deal 


\begin{tabular}{ccc}
\hline Category & Attributes & Verb \\
\hline Batting & 9 & 47.0 \\
Bowling & 11 & 10.2 \\
Fall of Wickets & 8 & 46.4 \\
Match Details & 11 & 75.2 \\
Match Result & 8 & 45.1 \\
Officials & 8 & 6.0 \\
Partnerships & 11 & 75.5 \\
Team Statistics & 13 & 46.2 \\
\hline
\end{tabular}

Table 1: Number of attributes per category with percent verbalised (Verb)

with this by enforcing a stronger structure - dividing the information into eight of our own "categories', based roughly on the formatting of the webpages. These are outlined in Table 1.

The first three categories in the table are quite intuitive and implicit from the respective sections of the scorecard. There is additional information in a typical scorecard (not shown in Figure 1), which we must also categorise. The 'Team Statistics' category contains details about the 'extras'3 scored by each team, as well as the number of points gained by the team towards that particular series ${ }^{4}$. We divide the remaining tag attributes as follows into three categories: 'Officials' - persons participating in the match, other than the teams (e.g. umpires, referees); 'Match Details' - information that would have been known before the match was played (e.g. venue, date, season); and 'Match Result' - data that could only be known once the match was over (e.g. final result, player of the match).

Finally we have an additional 'Partnerships' ${ }^{5}$ category which is given explicitly on a separate webpage referenced from each scorecard, but is also implicit from information contained in the 'Fall of Wickets' and 'Batting' sections. We anticipate that this category will help us manage the issue of data sparsity. For instance, in our domain we could group partnerships (which could contain a multitude of player combinations and there-

\footnotetext{
${ }^{3}$ Additional runs awarded to the batting team for specific actions executed by the bowling team. There are four types: No Ball, Wide, Bye, Leg Bye.

${ }^{4}$ Each cricket game is part of a specific 'series' of games. e.g. India would receive five points for their win within the NatWest series.

${ }^{5} \mathrm{~A}$ 'partnership' refers to a pair of players who bat together, and usually comprises information such as the number of runs scored between them, the number of deliveries faced and so on.
}

fore distinct tags) with the various possible binary combinations of players together for shared learning. We discuss this further in Section 8.3.

Within 5 of the categories described above, we are further able to divide the data into 'groups' the Batting, Bowling, Fall of Wickets and Partnerships categories refer to multiple players and thus have multiple rows. The Team Statistics category contains two groups, one for each team. The other categories merely form one-line tables.

\section{Machine Learning}

Our task is to establish which tag attributes (and hence tag values) should be included in the final match report, and is a multi-label classification problem. We chose to use BoosTexter (Schapire and Singer, 2000) as it has been shown to be an effective classifier (Yang, 1999), and it is one of the few text classification tools which directly supports multi-label classification. This is also what B\&L used.

Schapire and Singer's BoosTexter (2000) uses 'decision stumps', or single level decision trees to classify its input data. The predicates of these stumps are defined, for text, by the presence or absence of a single term, and, for numerical attributes, whether the attribute exceeds a given threshold, decided dynamically.

\subsection{Running BoosTexter}

BoosTexter requires two input files to train a hypothesis, 'Names' and 'Data'.

Names The Names file contains, for each possible tag attribute, $t$, across all scorecards, the type of its corresponding tag value. These are continuous for numbers and text for normal text. From our 133 scorecards we extracted a total of 61,063 tag values, of which $82.2 \%$ were continuous, the remainder being text.

Data The Data file contains, for each scorecard, a comma-delimited list of all tag values for a particular scorecard, with a '?' for unknown values, followed by a list of the verbalised tag attributes.

Testing We can now run BoosTexter with a user-defined number of rounds, $T$, which creates a hypothesis file. Using this hypothesis file and a test 'data' file (without the list of verbalised tag attributes), BoosTexter will give its hypothesized predictions, a value $f$ for each tag attribute $t$. The sign of $f$ determines whether the classifier believes the tag value corresponding to $t$ is relevant 
to the test scorecard, while $|f|$ is a measure of the confidence the classifier has in its assertion.

\subsection{Data Sparsity}

The very nature of the data means that there are a large number of tag values which do not occur in every scorecard - the average scorecard contained 24 values, yet our 'names' file contained 1193 possible tag attributes. A lot of this was due to partnership tag attributes which formed $43.6 \%$ of the 'names' entries. This large figure is because a large number of all possible binary combinations of players existed in the training data across both teams ${ }^{6}$. This implies we will be unable to train for a significant number of tag attributes as many specific tag values occur very rarely. Indeed we found that of 158,669 entries, 97,666 (61.55\%) were 'unknown'.

\section{Evaluation Baselines}

It is not clear what constitutes a suitable baseline so we considered multiple options. The issue of ambiguous reference baselines is not specific to the cricket domain, as there is no standardized baseline approach across the prior literature. We employ ten-fold cross validation throughout.

\subsection{Majority Baseline}

B\&L created a 'majority baseline' whereby they returned those categories (i.e., tables) which were verbalised more than half of the time in their aligned reports.

As explained in Section 3.2 we divided our tag attributes into 8 categories. We emulated B\&L's baseline method as follows: For each category, if any of the tag values within a particular 'group' was tagged as verbalised, we counted that as a 'vote' for that particular category. We then calculated the total number of 'votes' divided by the total number of 'groups' within each category. All categories which had a ratio of $50 \%$ or greater in this calculation were considered to be 'majority categories'. Our baseline $B_{\text {maj }}$ then consisted of all tag attributes forming part of those majority categories. As shown in Table 1 there were only two categories which exceeded the 50\% threshold, 'Match Details' and 'Partnerships'.

We can see that this baseline performs abysmally. The reason for this poor behaviour is

\footnotetext{
${ }^{6} 93$ of the possible $2 \sum_{i=1}^{10} i=110$ combinations oc-
}

\begin{tabular}{ccccc}
\hline$B_{\text {maj }}$ & $\mu$ & $\min$ & $\max$ & $\sigma$ \\
\hline Precision & 0.0966 & 0.0333 & 0.1583 & 0.0250 \\
Recall & 0.4879 & 0.2727 & 0.7895 & 0.0977 \\
F & 0.1603 & 0.0620 & 0.2568 & 0.0384 \\
\hline
\end{tabular}

Table 2: Majority Baseline, $B_{\text {maj }}$

that since so many tag attributes contribute to the categories we are including far too many possibilities in our baseline.

\subsection{Probabilistic Baseline}

This baseline is based on the premise that those tag attributes which occur with highest frequency across the training data refer to those tag values which will often occur in a typical match report. To create our baseline set of tag attributes $B_{\text {prob }}$ we extract the $a$ most frequently verbalised tag attributes across all the training data where $a$ is the average length of the verbalised tag attribute lists for each report/scorecard pair.

\begin{tabular}{ccccc}
\hline$B_{\text {prob }}$ & $\mu$ & $\min$ & $\max$ & $\sigma$ \\
\hline Precision & 0.5157 & 0.2174 & 0.7391 & 0.1010 \\
Recall & 0.5157 & 0.1389 & 0.7647 & 0.0990 \\
F & 0.5100 & 0.1695 & 0.6939 & 0.0852 \\
\hline
\end{tabular}

Table 3: Probabilistic Baseline, $B_{\text {prob }}$

This baseline achieves a mean F score of $51 \%$, however the tag attributes being returned are very inconsistent with a typical match report - they correspond in the majority to player names but not one refers to any other tag attributes relevant to those players. This renders the output mostly meaningless in terms of our aim to select content for an NLG system.

\subsection{No-Player Probabilistic Baseline}

Taking the above into account we create a baseline which derives its choice of tag attributes from match statistics only. This baseline is similar to the Probabilistic Baseline above, with the exception that when summing the numbers of tag attributes in the sets we do not consider player-name tag attributes in our counts. Instead, we extract the $a^{\prime}$ most frequent tag attributes, where $a^{\prime}$ is the average size of the sets excluding player-name tag attributes. To finally obtain our baseline set $B_{\text {nops }}$ we merge our $a^{\prime}$ most frequent tag attributes 
with any and all corresponding player-name tag attributes ${ }^{7}$.

\begin{tabular}{ccccc}
\hline$B_{\text {nops }}$ & $\mu$ & $\min$ & $\max$ & $\sigma$ \\
\hline Precision & 0.4923 & 0.1765 & 0.6875 & 0.0922 \\
Recall & 0.3529 & 0.1111 & 0.5625 & 0.0842 \\
F & 0.4064 & 0.1538 & 0.5946 & 0.0767 \\
\hline
\end{tabular}

Table 4: No-Player Probabilistic Baseline, $B_{\text {nops }}$

As can be seen from Table 4, this method suffers an absolute F-score drop of more than $10 \%$ from the previous method. However if we analyse the output more closely we can see that although the accuracy has dropped, the returned tag attributes are more thematically consistent with the training data. This is our preferred baseline.

\section{Evaluation Paradigm}

The main difficulty we encountered arose when we came to assessing the Precision and Recall figures as we have yet to decide on what level we are considering the output of our system to be correct. We see three possibilities for the level:

Category We could simply count the 'votes' predicted on a per category basis (as described in sections 3.1 and 5.1), and evaluate categories based on the number of votes given for each. We would expect this to generate very good results as we are effectively overgrouping, once on a group basis (grouping together all attributes) and once on a category basis (unifying all groups within a category), but the output would be so general and trivial (effectively stating something to the effect that "a match report should contain information about batting, bowling and team statistics") that it would be of no use in an NLG system.

Groups Here we compare which 'groups' were verbalised within each category, and which were predicted to be verbalised (as we did for the Majority Baseline of Section 5.1). Our implicit grouping means that we do not have to necessarily return the correct statistic pertaining to a group since each group acts as a basket for the statistics contained within it, and is susceptible to 'false positives'. This method is most similar to B\&L's.

Tags Since we are trying to establish which tag attributes should be included rather than which groups are likely to contain verbalised tag attributes, we could say that even the above method

\footnotetext{
${ }^{7}$ e.g., if team 1_player4_R is in $a^{\prime}$ then we would also include team1 player4 in our final set.
}

is too liberal in its definition of correctness. Thus we also evaluate our groups on the basis of their component parts, i.e., if a particular group of tag attributes is estimated to be verbalised by BoosTexter, then we include all attributes from that group.

\section{Initial Results}

Our 'categorized' results are derived from presenting BoosTexter with each individual category as described in Section 3.2, then merging the selected tag attributes together and evaluating based on the criteria described above. We then show BoosTexter's performance 'as is', by running the program on the full output of our alignment stage with no categorization/grouping.

\subsection{Categorized - Groups Level}

Our 'Categorized Groups' results can be found in Figure 3 and Table 5. For each of our tests we vary the value of $T$ (the number of rounds) to see how it affects our accuracy.

Here we see we have a maximum $\mathrm{F}$ score of 0.7039 for $T=25$. This is a very high result, performing far better than all our baselines, however we feel the 'basketing' mentioned in Section 6 means that the results are not particularly instructive - instead of specific 'interesting' tag attributes, we return a grouped list of tag attributes, only some of which are likely to be 'interesting'. Thus we decide to no longer pursue 'grouping' as a valid evaluation method, and evaluate all our methods at the 'tag attribute' level.

\begin{tabular}{ccccc}
\hline & & Best & $\mu$ & $\sigma$ \\
\hline \multirow{3}{*}{ CG } & Precision & 0.7620 & 0.7473 & 0.0320 \\
& Recall & 0.6795 & 0.6680 & 0.0322 \\
& F & 0.7039 & 0.6897 & 0.0106 \\
\hline
\end{tabular}

Table 5: Categorized Groups with Best value for $T=25$.

\subsection{Categorized - Tags Level}

What is notable here is that, for all values of $T$ which we ran our tests on (ranging from 1 to 3000), we obtained just one set of results for 'Categorized Tags', displayed in Table 6.

This behaviour indicates that the boosting is not helping to improve the results. Rather, it is repeatedly producing the same hypotheses, with varying confidence levels. The low F score is due to 


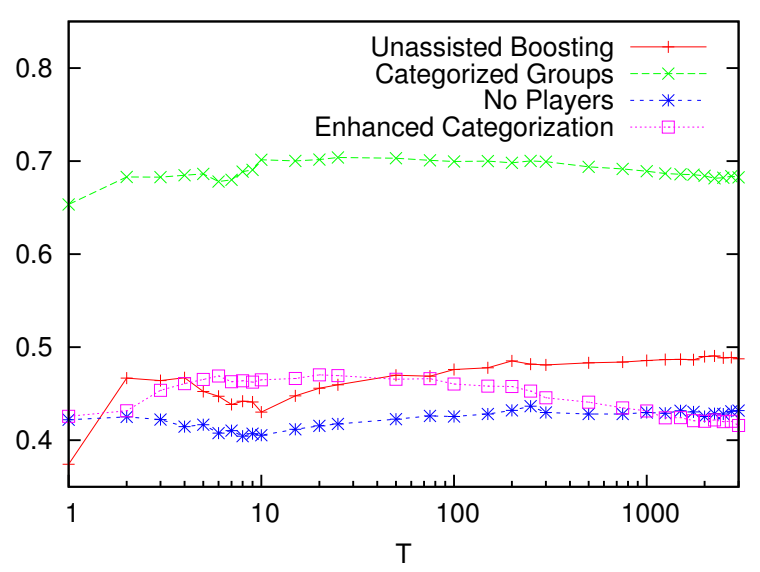

Figure 3: All F scores Results

\begin{tabular}{ccccc}
\hline & $\mu$ & $\min$ & $\max$ & $\sigma$ \\
\hline Precision & 0.0880 & 0.0496 & 0.1933 & 0.0223 \\
Recall & 0.7872 & 0.5417 & 1.0000 & 0.1096 \\
F & 0.1575 & 0.0924 & 0.3151 & 0.0361 \\
\hline
\end{tabular}

Table 6: Categorized Tags Results

the very low Precision value. This method is effectively a direct application of B\&L's method to our domain, however because of our strict accuracy measurement, it does not perform particularly well. In fact it is even worse than $B_{\mathrm{maj}}$, our worstperforming baseline. We believe this is because the Majority Baseline is limited in the breadth of tags returned, whereas this method returns very large sets of over 200 tag attributes (due to the many contributing tag attributes of each category) while the average size of the training sets is 24 .

Ideally we want to strike a balance between the improved granularity of the Categorized Tags evaluation (without the low accuracy) with the excellent performance of the Categorized Groups evaluation (without the too-broad basketing).

\subsection{Unassisted Boosting}

Our results are in Table 7 (row UB) and Figure 3. We can see $F$ values are increasing on the whole, and that we have nearly reached our Probabilistic Baseline. Inspecting the contents of the sets returned by BoosTexter, we see they are slightly more in line with a typical training set, but still suffer from an over-emphasis on player names. We also believe the high number of rounds required for our best result $(T=2250)$ is caused by the sparsity issue described in Section 4.2.

\begin{tabular}{ccccc}
\hline & & Best & $\mu$ & $\sigma$ \\
\hline \multirow{4}{*}{ UB } & Precision & 0.4965 & 0.4730 & 0.0253 \\
& Recall & 0.4961 & 0.4723 & 0.0252 \\
& F & 0.4907 & 0.4673 & 0.0249 \\
\hline \multirow{4}{*}{ NP } & Precision & 0.4128 & 0.3976 & 0.0094 \\
& Recall & 0.4759 & 0.4633 & 0.0126 \\
& F & 0.4367 & 0.4227 & 0.0091 \\
\hline \multirow{2}{*}{ EC } & Precision & 0.4440 & 0.4318 & 0.0136 \\
& Recall & 0.5127 & 0.4753 & 0.0271 \\
& F & 0.4703 & 0.4467 & 0.0194 \\
\hline
\end{tabular}

Table 7: Unassisted Boosting (UB), No Players (NP) and Enhanced Categorization (EC). Best values for $T=2250,250$ and 20 respectively.

\section{No-Players \& Enhanced Categorization}

We now consider alternative, novel methods for improving our results.

\subsection{Player Exclusion}

We have thus far ignored coherency in our data - for example we want to make sure that player statistics will be accompanied by their corresponding player name.

One problem so far with our approach has been that we are effectively double-counting the players. Our methods inspect which player names should appear at the same time as finding appropriate match statistics, whereas we believe we should instead be finding relevant statistics in the first instance, holding back player names, then including only those players to whom the statistics refer. Thus we restate our task in this way.

This is also sensible as in previous incarnations the learning algorithm had been learning from the literal strings of the player names. Although a player could be more likely to be named for various reasons, these reasons would not appear in the scorecard and we feel the strings are best ignored.

Thus we decide to remove all player names from the machine learning input, reinstating only relevant ones once BoosTexter has selected its chosen tag attributes.

\subsection{Player Exclusion Results}

As can be seen from Table 7 (row NP) and Figure 3 , we have a maximum $\mathrm{F}$ value of 0.4367 when $T=250$, and have achieved a $3 \%$ absolute increase, over our $B_{\text {nops }}$ baseline, a static implementation of the above ideas. 


\subsection{Enhanced Categorization}

Our final method combines the ideas of Section 8.1 above with the benefits of categorization, and handles data sparsity issues.

The method is identical to that of Section 3.1, with two important exceptions: The first is that we reintroduce player names after the learning, as above. The second is that instead of just a binary include/don't-include decision for each tag attribute, we offer a list of verbalised tag attributes to the learner, but anonymising them with respect to the group in which they appear. This enables the learner to, given any group, predict which tag attributes should be returned, independent of the group in question. This means groups with oftenempty tag values are able to leverage the information from groups with usually populated tag values, hence solving our data-sparsity issues. For example, this will solve the issue, referenced in Section 4.2 of a lack of training data for particular player-combination partnerships.

Having held back the group to which the tag attributes belong, we reintroduce them enabling discovery of the original tag attribute. This offers the benefits of categorization, but with a finer-grained approach to the returned sets of tag attributes.

\subsection{Enhanced Categorization Results}

Our results are in Table 7 (row EC) and Figure 3 . We achieved our best $F$ score result of 0.4703 for a relatively low value of $T=20$, and we can clearly see that boosting establishes a reasonable ruleset after a small number of iterations - we believe we have resolved the issue of data sparsity. The fact that this grouping has improved our results compared to feeding the information in 'flat' (as in Section 7.3) emphasises that the construction and make-up of the categories play a key role in defining performance.

\section{Conclusions \& Future Work}

This paper has presented an exploration of various methods which could prove useful when selecting content given a partially structured database of statistics and output text to emulate. We began by acquiring the necessary domain data, in the form of scorecards and reports, and employed a six-step process to align scorecard statistics verbalised in the reports. We next categorised our statistics based on the scorecard format. We established three baselines - one 'unthinking' proba- bilistic baseline, a 'sensible' probabilistic one, and another using categorization.

We found that unassisted boosting actually performed worse than our comparable probabilistic baseline, $B_{\text {prob}}$, but its output was marginally more in line with the typical training data. We explored how categorization affected our results, and showed that by grouping similar sets of tag attributes together we achieved a $7.4 \%$ improvement over the comparable baseline value, $B_{\text {nops }}$ (Table 4). We further improved this technique in a novel way by sharing structural information between learning instances, and by holding back certain information from the learner. Our final best Fvalue marked a relative $15.7 \%$ increase on $B_{\text {nops }}$.

There are multiple avenues still available for exploration. One possibility would be to further investigate the effects of categorization from Section 3.2 , for example by varying the size and number of categories. We would also like to apply our methods to another domain (e.g. rugby games) to establish the relative generality of our approach.

\section{Acknowledgments}

This paper is based on Colin Kelly's M.Phil. thesis, written towards his completion of the University of Cambridge Computer Laboratory's Computer Speech, Text and Internet Technology course. Grateful thanks go to the EPSRC for funding.

\section{References}

Regina Barzilay and Mirella Lapata. 2005. Collective Content Selection for Concept-To-Text Generation. In HLT '05, pages 331-338. Association for Computational Linguistics.

Jose Coch. 1998. Multimeteo: multilingual production of weather forecasts. ELRA Newsletter, 3(2).

Cricinfo. $2007 . \quad$ Wisden Almanack. http://cricinfo.com/wisdenalmanack. Retrieved 28 April 2007. Registration required.

Pablo A. Duboue and Kathleen R. McKeown. 2003. Statistical Acquisition of Content Selection Rules for Natural Language Generation. EMNLP ‘03, pages 121-128.

Ehud Reiter and Robert Dale. 2000. Building Natural Language Generation Systems. Cambridge University Press.

Jacques Robin. 1995. Revision-based generation of natural language summaries providing historical background: corpus-based analysis, design, implementation and evaluation. Ph.D. thesis, Columbia University.

Robert E. Schapire and Yoram Singer. 2000. BoosTexter: A boosting-based system for text categorization. Machine Learning, 39(2/3):135-168.

Yiming Yang. 1999. An evaluation of statistical approaches to text categorization. Information Retrieval, 1(1/2):6990 . 